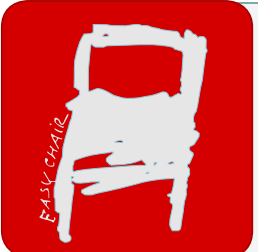

EPiC Series in Health Sciences

CAOS 2018. The 18th Annual Meeting of the International Society for Computer Assisted Orthopaedic Surgery

\title{
Quantification of Impingement-Free Range-of-Motion in Reverse Shoulder Arthroplasty Planning
}

Katrien Plessers $\mathrm{MSc}^{1 *}$, Roel Wirix-Speetjens $\mathrm{PhD}^{2}$, Ilse Jonkers $\mathrm{PhD}^{3}$, Philippe Debeer $\mathrm{PhD}, \mathrm{MD}^{4}$, Jos Vander Sloten $\mathrm{PhD}^{5}$

1,5*Biomechanics Section, KU Leuven, Leuven, 3000, Belgium, katrien.plessers@kuleuven.be

${ }^{2}$ Materialise N.V., Heverlee, 3001, Belgium

${ }^{3}$ Departement of kinesiology, KU Leuven, Leuven, 3000, Belgium

${ }^{4}$ Orthopaedics, University Hospitals Leuven, \& Dep. of Development and Regeneration, KU Leuven \& Institute for Orthopaedic Research and Training, Leuven, 3000, Belgium

\section{INTRODUCTION}

Bony impingement is a common complication in reverse shoulder arthroplasty (RSA). Since its occurrence depends on patient anatomy and glenoid implant position, surgeons need to be aware of it when preoperatively planning an implant (Gutiérrez 2008, Li 2013, Smith 2015). Currently, methods exist to evaluate the impingementfree range-of-motion (ROM) during preoperative planning (Krekel 2009, Roche 2013). However, these methods do not result in one overall score, which is required for an objective comparison. Therefore, our study aims to quantify the impingementfree ROM in one objective score so that surgeons can easily optimize the implant position for each patient.

\section{MATERIALS AND METHODS}

In this work, we evaluate the impingement-free ROM by computing the extreme poses of the humeral liner and mapping them as a curve on a two-dimensional (2D) polar plot (Figure 1). To quantify the result, we define a clinically relevant area on the polar plot, based on healthy kinematic motion data (Ludewig 2009). A ROM score is then defined as the percentage of the clinically relevant area that lies within the 
impingement-free ROM curve. Using this method, a 100\% ROM score means that the full clinically relevant area can be reached by the humeral liner.

To evaluate the performance of this ROM scoring method, we created 250 virtual scapula shapes using a statistical shape model (SSM) of 66 healthy scapulae. First, 50 random scapula shapes were generated to investigate the effect of glenoid implant positioning. Then, to study the effect of scapula shape variations, the first and second shape coefficient of these 50 random scapula shapes were subsequently set to $-2 \sigma$ and $+2 \sigma$, with $\sigma$ the standard deviation of the shape coefficient distribution. This resulted in an additional 200 scapula shapes in which each shape had its first or second mode fixed to $-2 \sigma$ or $+2 \sigma$ (Fig 2).

Finally, the 250 scapula shapes were virtually implanted with a baseplate and a $42 \mathrm{~mm}$ glenosphere (Delta Xtend, Depuy Synthes) in a fixed position with respect to the glenoid center point. In the 50 random scapula shapes, additional implants were planned with a $5 \mathrm{~mm}$ variation in the medial, lateral, inferior, superior, anterior and posterior direction. For every planned implant, we evaluated the impingement-free ROM using a $6 \mathrm{~mm}$ thick humeral liner.
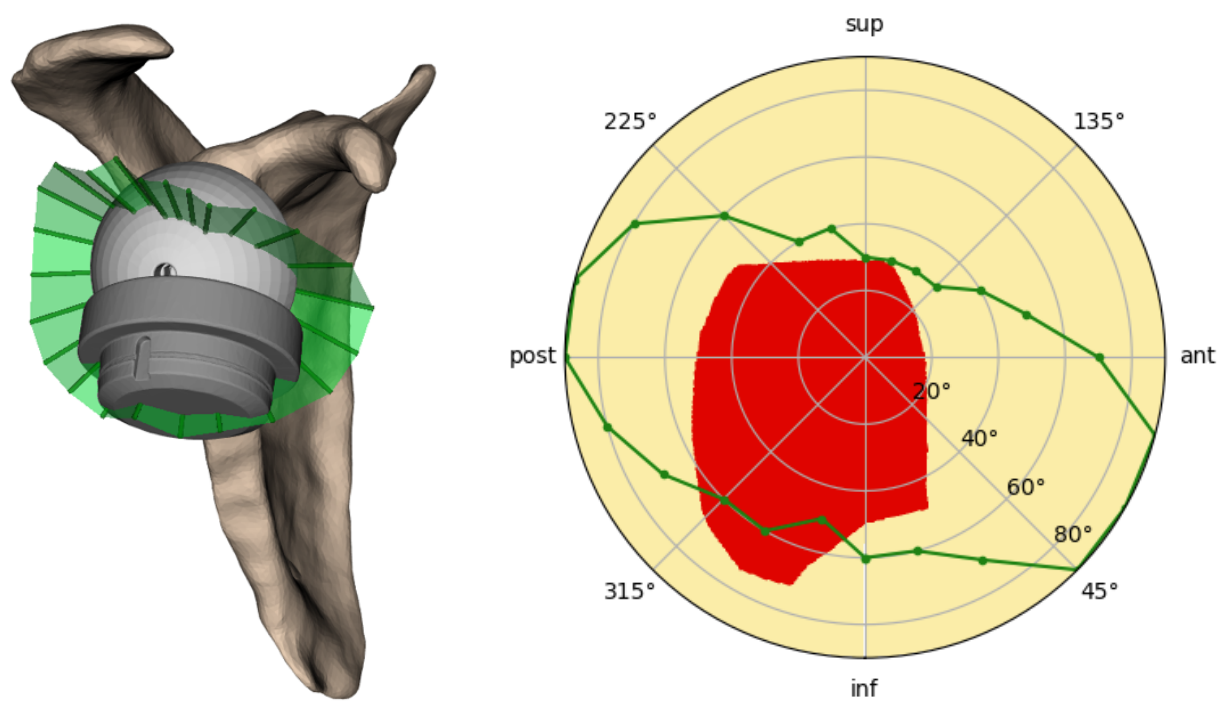

Figure 1: (left) The green umbrella shows the impingement-free ROM, as confined by the humeral liner. (right) The impingement-free ROM is mapped as a closed curve (green) on a 2D polar plot. A clinically relevant area (red) is defined based on healthy kinematic motion data.

\section{RESULTS}

ROM scores of the 50 random scapula shapes with an implant in the neutral position have a mean value of $0.84+/-0.06$. A lateral, inferior and posterior implant position improved the mean ROM score to respectively $0.93+/-0.04,0.90+/-0.05$ and 0.88 
+/- 0.05 (Fig 2). A medial, superior and anterior implant position showed the reverse effect. Moreover, a negative shape coefficient in the first mode and a positive shape coefficient in the second mode increased the ROM scores towards a mean value of $0.89+/-0.04$ and $0.90+/-0.04$ respectively. The effects of implant planning and scapula shape on the ROM were also visible on the 2D polar plots (Fig 2). The plots showed that the inferior portion of the clinically relevant area was often not reachable.

\begin{tabular}{|l|l|l|l|}
\hline $\begin{array}{l}\text { Shape } \\
\text { coefficients }\end{array}$ & $\mathbf{N}$ & $\begin{array}{l}\text { Implant } \\
\text { position }\end{array}$ & $\begin{array}{l}\text { Mean } \\
\text { ROM score }\end{array}$ \\
\hline Random & 50 & Neutral & $0.84+/-0.06$ \\
\hline Random & 50 & Medial $5 \mathrm{~mm}$ & $0.71+/-0.07$ \\
\hline Random & 50 & Lateral $5 \mathrm{~mm}$ & $0.93+/-0.04$ \\
\hline Random & 50 & Superior $5 \mathrm{~mm}$ & $0.69+/-0.08$ \\
\hline Random & 50 & Inferior $5 \mathrm{~mm}$ & $0.90+/-0.05$ \\
\hline Random & 50 & Anterior $5 \mathrm{~mm}$ & $0.72+/-0.11$ \\
\hline Random & 50 & Posterior $5 \mathrm{~mm}$ & $0.88+/-0.05$ \\
\hline$-2 \sigma$ in mode 1 & 50 & Neutral & $0.89+/-0.04$ \\
\hline $2 \sigma$ in mode 1 & 50 & Neutral & $0.71+/-0.08$ \\
\hline$-2 \sigma$ in mode 2 & 50 & Neutral & $0.76+/-0.06$ \\
\hline $2 \sigma$ in mode 2 & 50 & Neutral & $0.90+/-0.04$ \\
\hline
\end{tabular}




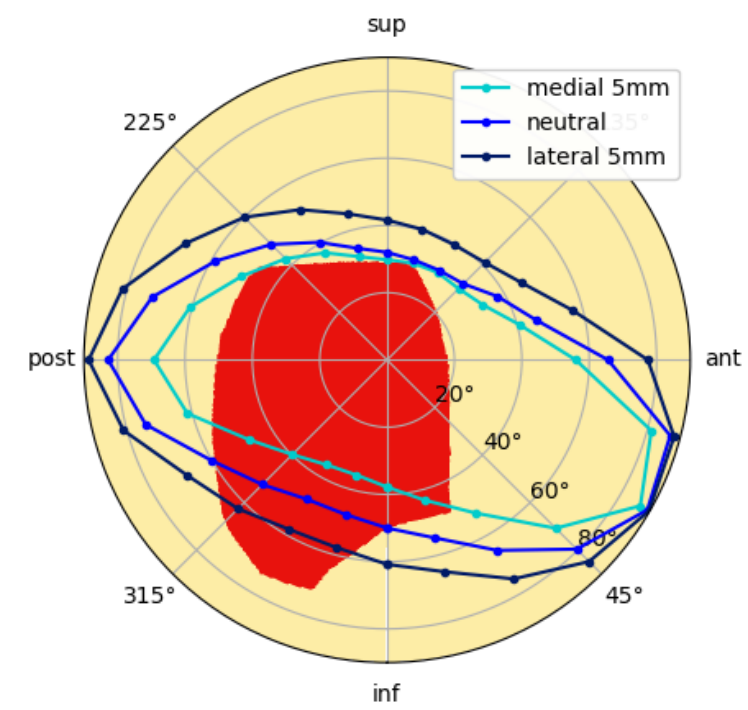

Figure 2: (left) Mean ROM scores indicating the effect of implant position and scapula shape. (right) The mean impingement-free ROM of random scapula shapes with an implant in the neutral, $5 \mathrm{~mm}$ medial and $5 \mathrm{~mm}$ lateral position. Lateralisation has a positive effect on the ROM since the humeral liner can reach a larger portion of the clinically relevant area.

\section{DISCUSSION}

Our study presented a method to quantify impingement-free ROM in one objective score, using healthy kinematic motion data. A lateral and inferior implant position resulted in a higher ROM score, which agrees with many studies in literature (Gutiérrez 2008, Li 2013). As the first shape mode corresponds to the size of the scapula, our study also indicates that smaller scapulae lead to better ROM scores. Due to the small shape of the glenoid face, the humeral liner has less chance to collide against it. Inferior impingement resulting in notching, is one of the major impingement occurrences and can be explained by the unreached inferior portion of the clinically relevant area. We conclude that the ROM score proposed in this study agrees well with what has been published in literature and is therefore considered as a suitable candidate to objectively evaluate and optimize the implant plan during preoperative planning.

\section{REFERENCES}

- Gutiérrez S, Comiskey CA, Range of Impingement-Free Abduction and Adduction Deficit After Reverse Shoulder Arthroplasty, J. Bone Jt. Surg., 90(12), pp. 2606 2615, 2008.

- Krekel PR, de Bruin PW, Evaluation of bone impingement prediction in preoperative planning for shoulder arthroplasty, Proc. Inst. Mech. Eng, 223(7), pp. $813-822,2009$. 
- Li X, Knutson Z, Effects of glenosphere positioning on impingement-free internal and external rotation after reverse total shoulder arthroplasty, J. Shoulder Elbow Surg., 22(6), pp. $807-813,2013$.

- Ludewig PM, Phadke V, Motion of the Shoulder Complex During Multiplanar Humeral Elevation, J. Bone Jt. Surg., 91(2), pp. 378 - 389, 2009.

- Roche CP, Marczuk Y, Scapular notching in reverse shoulder arthroplasty: validation of a computer impingement model, Bull. Hosp. Jt. Dis., 71(4), pp. 278 $-283,2013$.

- Smith T, Bäunker A, Biomechanical evaluation of inferior scapula notching of reverse shoulder arthroplasty depending on implant configuration and scapula neck anatomy. Int. J. Shoulder Surg., 9(4), pp. 103-109, 2015.

\section{DISCLOSURES}

Katrien Plessers and Roel Wirix-Speetjens are employees of Materialise N.V., but this relationship has no bias on their work and did not inappropriately influence this study.

Philippe Debeer works as a consultant for Materialise N.V., but this relationship has no bias on his work and did not inappropriately influence this study. 